\title{
EL PROCESO DE ENFERMAR DE LOS MÉDICOS Y SUS CONSECUENCIAS EN EL PROFESIONALISMO
}

\author{
Bárbara Marco-Gómez ${ }^{1}$, Candela Pérez-Alvárez ${ }^{1}$, Alba Gallego-Royo ${ }^{1}$, Teresa Martínez-Boyero ${ }^{1}$, Ro- \\ gelio Altisent ${ }^{1}$, Teresa Delgado-Marroquín ${ }^{1}$, María Pilar Astier-Peña ${ }^{1}$
}

Resumen: La idiosincrasia de la profesión médica, los rasgos de personalidad característicos del médico y la falta de formación específica para reconocer y tratar adecuadamente la propia vulnerabilidad, predisponen a este colectivo a padecer más patología mental y, probablemente, un deficiente tratamiento de otras enfermedades. La mayoría de los estudios realizados hasta el momento se centran en la patología mental y adictiva del médico, desde el punto de vista del riesgo para la mala praxis y la seguridad de sus pacientes. La revisión narrativa de la literatura científica realizada (MEDLINE, EMBASE e IME 19852016) ha mostrado que, en el entorno hispanoparlante, apenas disponemos de información actualizada sobre el médico como paciente, a pesar de ser un tema de indudable relevancia desde el punto de vista de la seguridad asistencial, del profesionalismo y del propio bienestar de los profesionales. La situación del médico que enferma es compleja y poco conocida, con conflicto de roles y con repercusiones en la ética profesional y la calidad asistencial.

Palabras clave: enfermedad, médico, estudiante de medicina, médico residente, jubilación, automedicación, autotratamiento, acceso a la atención, programas de salud, salud mental, ética médica, deontología profesional

\section{The process of physicians falling ill and consequences for their professionalism}

\begin{abstract}
The idiosyncrasy of the medical profession, the characteristic personality traits of the physician and the lack of specific training to properly recognize and treat one's own vulnerability dispose this group to suffer more mental pathology and probably worse control of other medical diseases. Most of the studies promoted so far focus on the psychiatric and addictive concerns of the physician, from the point of view of the sick doctor as being at risk for malpractice and to guarantee the safety of their patients. The narrative review of scientific literature (MEDLINE, EMBASE and IME 1985-2016) has shown that we do not have in the Spanish-speaking environment updated information about the doctor as a patient, despite being a topic of incipient media repercussion and undoubtedly relevant from the point of view of patient safety, professionalism and medical ethics and as well as the welfare of professionals. The characteristic of a sick doctor are more complex than the rest of patients with a conflict of roles and repercussions on professional ethics and the quality of care.
\end{abstract}

Key words: illness, physician, medical student, junior physician, retirement, self-prescription, self-treatment, access to health care, health plan, mental health, medical ethics, professionalism

\section{O processo de adoecimento dos médicos e as suas consequências acerca do profissionalismo}

Resumo: A idiossincrasia da profissão médica, as características de personalidade característica do médico e a falta de formação específica para reconhecer e, corretamente, tratar a própria vulnerabilidade, predispóem esse grupo a sofrer mais patologias mentais, e provavelmente tratamento deficiente de outras doenças. A maioria dos estudos realizados até agora tem como foco a patologia mental e o comportamento aditivo do médico, do ponto de vista do risco por negligência e segurança de seus pacientes. A revisão narrativa da literatura científica realizada (MEDLINE, EMBASE e IME 1985-2016) tem mostrado que no ambiente de idioma hispânico temos apenas informaçôes atualizadas sobre o médico como paciente, apesar de ser uma questão de relevância inquestionável do ponto de vista da segurança assistencial, do profissionalismo e do próprio bem-estar dos profissionais. A situação do médico enfermo é complexa e pouco conhecida, com conflito de papéis e impacto sobre a ética profissional e a qualidade dos cuidados médicos. São necessários mais estudos, tanto quantitativos como qualitativos, que permitam compreender o processo do adoecimento dos médicos em cada um dos seus estágios profissionais (desde a graduação até a aposentadoria) para ser capaz de considerar estratégias para a melhoria na atenção da saúde desses profissionais.

Palavras-chave: doença, médico, estudante de medicina, médicos residentes, aposentadoria, automedicação, auto tratamento, acesso a cuidados de saúde, saúde mental, ética médica, deontologia profissional

\footnotetext{
${ }^{1}$ Grupo de Investigación en Bioética, Fundación Instituto de Investigación Sanitaria de Aragón (IIS Aragón). Cátedra de Profesionalismo y Ética Clínica, Universidad de Zaragoza, España.

Correspondencia: mpastier@gmail.com
} 


\section{Introducción}

El hecho de enfermar es un proceso que tiene gran impacto en la vida personal y laboral, afectando a la esfera psicológica y volviendo vulnerable al individuo $(1,2)$. Cuando el que enferma es un médico, el proceso se torna más complejo, ya que instintivamente el profesional trata de dar solución por sí mismo al problema, poniendo tratamiento de forma rápida. Adquirir la condición de paciente le genera al profesional una situación de incongruencia interna, puesto que él mismo ha sido formado para diagnosticar, curar o paliar, pero no para abordar esta situación vital en su persona, ya que durante la formación del Grado no se plantea nunca el tema y, una vez en el ámbito laboral, los profesionales tienen interiorizado que solo pueden estar enfermos los pacientes.

Este conflicto interno entre el profesional que sana y la persona que enferma no se resuelve fácilmente. Los factores que influyen en la actitud de los médicos ante su propia salud y la de sus familiares abarcarían diferentes aspectos que se relacionan con su condición de médico como: sentirse como un experto en la enfermedad que diagnostican, estar familiarizado con los medios de diagnóstico y de tratamiento, conocer el ambiente y el funcionamiento de los centros sanitarios, y, en numerosos casos, la falta de apreciación de los límites de sus conocimientos.

Además, a esta situación se añade el hecho de que los circuitos asistenciales que utilizan los médicos cuando se sienten enfermos suelen ser atípicos, fuera de los procedimientos establecidos para los pacientes.

Por otro lado, tampoco los médicos que son consultados por otros colegas por problemas de salud actúan, en muchas ocasiones, como terapeutas profesionales y no saben establecer una adecuada relación médico paciente. Así, nos encontramos ante la paradoja de que los médicos, a pesar de ser expertos en la materia, cuando enferman no suelen utilizar los servicios médico-sanitarios y, cuando lo hacen, es de una forma muy inadecuada y desordenada.

Estas situaciones anómalas tienen impacto directo en la vida profesional del médico, tanto desde el punto de la pérdida de competencia profesional, como en el impacto de esta pérdida en la calidad de la atención que otorga a los pacientes que atiende, ya que la calidad asistencial de las instituciones sanitarias y sus resultados dependen directamente de la labor de sus profesionales. Por tanto, el profesionalismo médico es clave en la calidad asistencial de nuestros sistemas de salud(3).

Como bien ha puesto de manifiesto Firth-Cozens(4), los médicos enfermos no atendidos suponen un riesgo para su salud y la de sus pacientes y, además, generan un sinfín de problemas en los ámbitos familiar y laboral.

Esta situación afecta a todas las etapas del desarrollo profesional médico, con problemas diferentes en cada una de ellas, siendo el grupo de médicos en formación especializada uno de los más expuestos a padecer problemas mentales y adictivos, por la situación de estrés y por la escasa madurez profesional, mientras que el grupo de médicos jubilados encuentra dificultades para ser reconocido como médico y ser atendido como compañero en las instituciones sanitarias.

No disponemos en España ni en otros países de habla hispana de información actualizada sobre el proceso de enfermar del médico, excepto aquella que hace referencia al programa PAIME (Programa de Atención Integral al Médico Enfermo, Espańa) (5) para trastornos mentales y adictivos que presta atención únicamente a profesionales en activo, quedando fuera esta atención los médicos jubilados.

El proceso de enfermar de los médicos es por tanto un tema de incipiente repercusión mediática y de indudable relevancia desde el punto de vista de la seguridad del paciente, porque un elemento fundamental es el bienestar de los profesionales que, siendo óptimo, prestan una atención de calidad y segura.

El grupo de investigación de la Cátedra de Profesionalismo y Ética Clínica de la Universidad de Zaragoza está desarrollando un proyecto de investigación con el objetivo de mejorar la comprensión del proceso de enfermar del médico desde su etapa de estudiante hasta la jubilación, identificando sus puntos críticos con el fin, en primer 
lugar, de promover una actualización conceptual en el ámbito de la ética y la deontología médica que oriente sobre las actitudes y los comportamientos profesionales. En segundo lugar, se trata de promover áreas de mejora para una atención de calidad los profesionales de la medicina.

El presente artículo describe el proceso de revisión sobre el médico enfermo realizado por el grupo de investigación, estructurando los resultados en las diferentes etapas profesionales.

\section{Metodología}

Se ha realizado una revisión narrativa(6) entre 1985 y 2016 sobre el proceso de enfermar de los médicos. Para ello se ha realizado una búsqueda en MEDLINE a través de PubMed, EMBASE e IME. La estrategia de búsqueda se limitó a los idiomas inglés y español. Se han usado como descriptores y estrategia de búsqueda los referidos en la tabla 1:
La búsqueda bibliográfica con estos términos ha permitido identificar un número reducido de trabajos, por lo que se ha completado la selección de documentos mediante búsqueda inversa, es decir, por el análisis de las referencias de los documentos seleccionados. Esto ha permitido identificar a su vez a los autores más relevantes en el tema y revisar sus publicaciones relacionadas. Se revisó también documentos de organizaciones gubernamentales y de profesionales a nivel nacional e internacional accesibles a través de sus páginas web.

En la revisión se ha constatado que no existen unos descriptores $\mathrm{MeSH}$ específicos sobre el médico enfermo que permitan realizar una búsqueda precisa.

La selección de los artículos la realizaron cinco investigadores del proyecto. Cada artículo ha sido revisado en cuanto a su idoneidad con la línea de investigación por al menos dos investigadores.

Tabla 1: Descriptores y estrategia de búsqueda desplegada.

\begin{tabular}{|c|c|}
\hline Descriptores en inglés & Descriptores en español \\
\hline $\begin{array}{l}\text { Doctor/physician, health professionals, health } \\
\text { personnel. }\end{array}$ & Médico, doctor, profesional de la salud \\
\hline $\begin{array}{l}\text { Medical student, Junior physician, Registrars, } \\
\text { Attending physician, Retirement }\end{array}$ & $\begin{array}{l}\text { Estudiante de medicina, médico residente, médico } \\
\text { adjunto, médico jubilado }\end{array}$ \\
\hline Ill/sick/disease/health & Enfermo, enfermedad, salud \\
\hline Sick leave & Baja laboral \\
\hline Impairment & Discapacidad \\
\hline Physician heal thyself & El médico se auto-cura \\
\hline Self-care, self-medication & Autocuidado, automedicación, autoprescripción \\
\hline Access to health care/ Health plan & Acceso a la atención sanitaria \\
\hline \multicolumn{2}{|c|}{ Estrategia de búsqueda para artículos en inglés y español: } \\
\hline Detalle de búsqueda planteada en título y abstract: & $\begin{array}{l}\text { ("heal thyself”[tiab] OR "sick physicians"[tiab] } \\
\text { OR "sick physician"[tiab] OR "ill physicians"[tiab] } \\
\text { OR “ill physician"[tiab]) OR ("Physicians"[MAJR] } \\
\text { AND (ill[All Fields] OR sick[All Fields])) AND } \\
\text { ("1985/01/01"[PDat] : "2017/09/01"[PDat]) }\end{array}$ \\
\hline Secuencia: & $\begin{array}{l}\text { Los demás descriptores de la búsqueda se han } \\
\text { incorporado de forma secuencial: physician por } \\
\text { doctor, health profesional; sick por ill o health o } \\
\text { self-care... sucesivamente }\end{array}$ \\
\hline
\end{tabular}




\section{Resultados y discusión}

Pasamos a organizar los resultados de las publicaciones más importantes atendiendo a las características generales del proceso de enfermar del médico y a las particularidades de sus diferentes etapas profesionales.

\section{Hablemos de cifras...}

La cuantificación del problema de la enfermedad del médico no es una cuestión fácil de determinar. Stanton y Caan ya señalaron la dificultad de responder a la pregunta: “¿Cuántos médicos enfermos hay?" (7).

Numerosos estudios científicos e informes de instituciones (la mayoría pertenecientes al mundo anglosajón) ofrecen unas cifras muy dispares e incluso contradictorias. Algunos estudios en Estados Unidos señalan que la salud física de los médicos es comparable a la de la población general, o incluso mejor si se trata de las mujeres médicos $(8,9)$. Hay datos estimativos de que los médicos tienen una tasa estándar de mortalidad por causa física más baja que el resto de la población $(10,11)$. Sin embargo, es un hecho demostrado que las tasas de suicidio entre profesionales de la medicina son muy superiores a las de otros colectivos de igual nivel socioeconómico $(12,13)$. En Europa, los países nórdicos son quienes más han investigado sobre este tema desde el punto de vista laboral(14).

En cuanto al entorno hispanoparlante apenas existen estudios sobre el estado de salud física y la percepción que los médicos tienen de su enfermedad. En España, el estudio más amplio sobre el tema es la Encuesta sobre salud, estilos de vida y condiciones de trabajo de los médicos en Cataluña, realizada por la Fundación Galatea(15). En ella se concluye que los médicos tienen una prevalencia de enfermedades crónicas similar al resto de la población de la misma clase social, excepto para diabetes y sobrepeso (los médicos varones presentan proporciones superiores) y trastornos mentales y adictivos.

La gran mayoría de los estudios descriptivos epidemiológicos se centran en patologías del ámbito de la salud mental, especialmente aquellas rela- cionadas con el consumo de tóxicos y trastornos psiquiátricos asociados(16-18), que suponen mayor riesgo de interferencia laboral y potencial impacto sobre los pacientes y el propio sistema de salud(19-22).

\section{Características generales del médico enfermo: un paciente difícil...}

Bruguera et al.(23) afirman que los profesionales sanitarios enfermos presentan aspectos diferenciales frente a otros grupos profesionales y la población general. Entre los factores que influyen en la actitud de los médicos ante su propia salud destaca el hecho de sentirse con el conocimiento experto de la enfermedad que padecen(24); conocer los medios diagnósticos y de tratamiento; conocer el ambiente y funcionamiento de los centros sanitarios, lo que lleva al uso de circuitos alternativos de atención (por ejemplo, consultas "de pasillo") (25); la falta de apreciación de los límites de sus conocimientos, que se relaciona con las elevadas tasas de autotratamiento $(26,27)$ y de incumplimiento de las indicaciones(28). A menudo surge la inclinación a negar y ocultar el problema, en lugar de buscar ayuda(29-32). Otras barreras que dificultan el acceso a la atención son la falta de confidencialidad, el miedo a perder la credibilidad ante los colegas y a la inhabilitación profesional o pérdida del puesto de trabajo(33). La negación y el miedo a la estigmatización también suponen un obstáculo para la reincorporación laboral(34).

De hecho, la autoprescripción es una característica diferencial clave entre los médicos y la población general, que merece una consideración particular y que ha sido bien estudiada por su gran prevalencia (80-90\%, dependiendo del estudio), su grado de aceptación (90\% de los médicos cree que es aceptable automedicarse en trastornos agudos)(35) y sus repercusiones. Esta práctica conlleva un retraso en la demanda formal de tratamiento y un mayor riesgo de iatrogenia. La automedicación tiene su justificación en el temor a la pérdida de la confidencialidad y el miedo al estigma de la enfermedad, especialmente la enfermedad mental, ya que se estima que más de dos tercios de los médicos con trastornos mentales se prescriben a sí mismos(36-38). No obstante, los medicamentos más frecuentemente autoprescritos, según Hem(39), son los antibióticos, se- 
guidos de los analgésicos y los hipnóticos. Esto podría sugerir una autoprescripción "cotidiana", basada en el sentimiento de autosuficiencia para tratar procesos leves y agudos, y acceso a realizar recetas para uno mismo y la familia.

Hay un acuerdo general en la literatura sobre la existencia de determinadas características comunes en la personalidad del médico que le hacen más vulnerable a un uso irregular del sistema. Firth-Cozens y Payne(40) las resumen en ocho: perfeccionismo excesivo y alto nivel de autoexigencia, elevadas aspiraciones de desarrollo profesional, aplazamiento continuo de las gratificaciones personales, escasos recursos personales para afrontar el estrés, hipocondriasis, narcisismo, autoestima vulnerable con excesiva dependencia de la valoración de los demás, y dificultades en las relaciones sociales.

\section{Las características del entorno laboral no ayu- dan...}

También hay que tener en cuenta la naturaleza demandante del trabajo desempeñado: la gran presión asistencial, la intensidad emocional y gran responsabilidad inherente al trato con enfermos, los recursos insuficientes, las guardias, la necesidad de actualización profesional constante... componen un caldo de cultivo ideal para el desarrollo de enfermedades médicas y psiquiátricas que bien podrían ser consideradas de origen laboral.

Otra cuestión es la actitud del equipo, que cambia ante un compañero enfermo, no reconocida abiertamente de dos formas casi opuestas pero igualmente contraproducentes: por un lado, haciendo una "conspiración del silencio" y resolviendo los problemas laborales o eventos adversos que puedan surgir, y tratando de cubrir al compañero enfermo con un falso concepto de respeto profesional; por otro, apartando al profesional enfermo del grupo sin abordar la situación, por desconocimiento de la forma adecuada de actuar, de tal manera que se acaba produciendo un deterioro del clima laboral y surgen conflictos con los compañeros(41).

\section{¿Cuál es el impacto en el proceso asistencial? La calidad y la seguridad del paciente}

Como pone de manifiesto Firth-Cozens(42), los médicos enfermos no atendidos suponen un riesgo para la salud de sus pacientes, ya que se produce una disminución de la competencia profesional con más errores(43), más confusiones, más solicitud de pruebas, más dudas; esto hace que la seguridad del paciente en el circuito asistencial se vea amenazada.

Las dificultades a la hora de asumir y tratar la enfermedad del médico repercuten también en términos económicos(44), ya que se producen bajas laborales de duración inadecuada, incapacidades permanentes e inhabilitaciones profesionales evitables, mayor número de demandas por mala praxis, etc.

La gran mayoría de los estudios en seguridad del paciente giran en torno a los incidentes o eventos adversos que sufren los pacientes (primera víctima), pero estos eventos adversos también son causa de alteraciones en la esfera laboral, familiar o personal de los profesionales involucrados, e impactan negativamente en la personalidad y la profesionalidad del médico(45). Gallagher et al.(46) encuestaron a una muestra de médicos canadienses y norteamericanos. De ellos, un 55\% reconoció haber cometido un error clínico con consecuencias graves para su paciente. Otros estudios (47) revelan que solo un 5\% del total de profesionales sanitarios se libra de experimentar de cerca o de forma directa un evento adverso (EA) a lo largo de su carrera profesional. En España, un $62 \%$ de profesionales de atención primaria y un $72,5 \%$ de profesionales en hospitales reconoce haber experimentado ser segunda víctima directamente, o bien referido a un colega próximo en los últimos cinco años(48).

El término "segunda víctima" hace referencia a los profesionales sanitarios que experimentan malestar emocional tras un evento adverso, malestar que se ha comparado al síndrome de estrés postraumático. Wu(49) define las segundas víctimas como los profesionales sanitarios implicados en un evento adverso que quedan traumatizados por esa experiencia o que no son capaces de afrontar emocionalmente la situación(50). 
Scott et al.(51-53) han descrito las consecuencias personales en las segundas víctimas, que se caracterizan por una respuesta de ansiedad, síntomas afectivos y preocupación mórbida por su rendimiento y capacidad profesional, que llega a afectar a su toma de decisiones clínicas. Éstas pueden presentar dificultades de concentración, frustración, culpa, síntomas depresivos y ansiedad, insomnio, ira y cambios de humor en el trabajo o en casa, dudas constantes sobre su capacidad profesional y miedo a consecuencias legales y pérdida reputación profesional(54). Es, por tanto, un problema de salud del profesional médico y que tiene repercusión en el sistema y que éticamente debe abordarse.

\section{El médico retirado sigue siendo médico}

La jubilación es un periodo de cambio significativo que requiere un esfuerzo de adaptación en cualquier persona $(55,56)$. Este acontecimiento tiene rasgos diferentes en el colectivo médico, debido a la idiosincrasia de la profesión y a las características de la personalidad del médico, ya comentadas(57).

Los factores más reseñables que condicionan el proceso de jubilación de los médicos son: el valor que el médico da en su vida a su profesión $(58,59)$, llegando a basar su identidad personal en la identidad profesional(60); la preocupación económica; la presencia de burnout(61) o el progresivo aumento de los problemas de salud asociados a la edad(62).

La jubilación del médico precisa de una adaptación y planificación, tal y como se muestra en varios estudios(63), proponiéndose la creación de opciones graduales o flexibles de jubilación y promocionando una cultura que facilite la participación de los médicos jubilados en la vida profesional, como consultores o como mentores de médicos en formación(64).

\section{La salud de los médicos residentes}

La residencia es un periodo formativo y, al mismo tiempo, supone la incorporación al mundo laboral y la práctica clínica(65). Se ha documentado que entre un 3 y un $10 \%$ de los médicos residentes tiene dificultades para alcanzar los ob- jetivos planteados en los programas de formación especializada(66). Estas dificultades pueden tener orígenes muy diversos, desde problemas de salud física o mental, o burnout, hasta dificultades de aprendizaje o situaciones estresantes a nivel personal $(67,68)$. El alto nivel de estrés parece tener relación con un peor estado de salud y una menor satisfacción vital(69).

Es complicado conocer datos epidemiológicos sobre los problemas de salud de los residentes. En España, los datos obtenidos del PAIME constatan un incremento de atenciones entre los médicos jóvenes $(70,71)$.

En los últimos años se ha incrementado el número de estudios sobre salud y calidad de vida en los residentes(72). Los principales temas estudiados han sido el síndrome de burnout(73,74), los trastornos del sueño, el estrés, la fatiga y las estrategias de afrontamiento(75). También se ha objetivado una prevalencia de depresión e ideación suicida más elevada que en la población general $(76,77)$.

Durante este periodo comienzan algunas conductas respecto de la propia salud, como la automedicación, cuya presencia durante la residencia puede predecir su existencia años después de titularse como especialista $(78,79)$.

El presentismo laboral (acudir a trabajar estando enfermo) es un fenómeno muy frecuente entre los médicos residentes (80). La presión por ir a trabajar estando enfermo puede ser particularmente importante en el caso de los médicos residentes, debido a la falta de una cobertura adecuada, las presiones ejercidas por los colegas o los superiores, miedo a no alcanzar la titulación de especialista, además de los sentimientos, comportamientos de sacrificio y compromiso adquiridos desde la universidad, y el miedo a ser considerado débil, siendo significativamente mayor en MIR del sexo femenino(81).

Desde su inicio en EE.UU. en los años ochen$\mathrm{ta}(82,83)$, se han creado múltiples programas de asistencia a médicos en formación a nivel internacional, sobre todo en el mundo anglosajón(84-86). Sin embargo, en España no existe aún un programa específico para residentes. 


\section{¿Cuándo se inicia el problema? El estudiante de medicina como punto de inflexión}

El profesional médico inicia su andadura en la esfera sanitaria adentrándose en el mundo universitario. El perfil del estudiante de medicina es el de un joven con altas cualificaciones y niveles muy altos de autoexigencia, que le permiten superar los exámenes necesarios para ser aceptado en una facultad de medicina. Este perfeccionismo excesivo, asociado a la escasez de recursos existentes para ayudar al afrontamiento del estrés y del fracaso, pueden confluir en una gran vulnerabilidad durante una época clave para el desarrollo profesional y personal(87).

El entorno en el que se mueve el estudiante parece ser el germen del inicio de trastornos depresivos y ansiosos, dando como resultado altas prevalencias de riesgo psicopatológico, en torno al $47 \%$ en comparación con la población de referencia(88). Además, las debilidades en la salud mental se incrementan conforme el estudiante va superando años de estudio(89).

Elementos como el burnout aparecen también en esta época, con una prevalencia entre el 45-71\%, y se observa en la literatura cómo puede estar relacionado con ideación autolítica en los estudiantes(90). En el estudio realizado por Dyrbye, un $11,2 \%$ de los estudiantes de las universidades estadounidenses había considerado el suicido en el último año, una cifra muy superior a la población de referencia $(6,9 \%)(91)$.

Respecto del consumo de drogas, se ha constatado un consumo de tranquilizantes muy superior en la población de estudiantes de medicina respecto de otros estudiantes universitarios.

Podría ser interesante llevar a cabo medidas para disminuir el estrés y aumentar el autocuidado de estos estudiantes, favoreciendo así la prevención de enfermedades en etapas profesionales posteriores, así como desarrollar, desde las facultades de medicina, actividades formativas para estudiantes, encaminadas a preparar al futuro profesional en la aceptación de su propia vulnerabilidad y capacitarle en el adecuado manejo tanto de su enfermedad como la de sus colegas.
Yel médico aprende de su propia enfermedad...

Los testimonios de los médicos que han padecido una enfermedad importante coinciden en señalar que vivir esa situación en primera persona les ha permitido mejorar su práctica clínica a través de un aumento de la empatía(92) con sus pacientes y una tendencia a proporcionar información más detallada sobre el proceso diagnóstico y terapéutico(93).

\section{¿Y qué ocurre con los "médicos de médicos"?}

Los médicos que son consultados por otros colegas encuentran dificultades a la hora de establecer una adecuada relación médico-paciente, puesto que en ocasiones reconocen al médico-paciente como paciente y, en otras, como un profesional competente(94). Según Thompson, existe una tendencia por parte de los médicos a reforzar una cultura que ignora el propio sufrimiento y el de sus colegas. De tal forma que no solo los médicos enfermos son reacios a pedir ayuda, sino que, cuando lo hacen, sus compañeros son reacios a intervenir o dan respuestas inadecuadas $(95,96)$.

\section{Programas de atención al médico enfermo}

En los años 70, en Estados Unidos(97), se crean los primeros programas de atención específica al médico enfermo, enfocados sobre todo al tratamiento de adicciones y trastornos mentales. Posteriormente se han ido desarrollando programas especiales dedicados a la salud de los profesionales sanitarios en todos los Estados de Estados Unidos(98) y en todas las provincias de Canadá(99), Australia y Nueva Zelanda(100). En cuanto a Europa, el British Medical Council del Reino Unido $(101,102)$ elaboró, durante la década de los 90, unas guías de actuación ante el médico enfermo.

En 1998 se creó en España el Programa de Atención Integral al Médico Enfermo (PAIME)(103), para el tratamiento de los médicos en activo con trastornos mentales y adictivos. Este programa da gran importancia a la confidencialidad y a la reinserción laboral(104). Siguen existiendo carencias en relación al manejo de otro tipo de trastornos médicos que también suponen una gran pérdida de calidad de vida, y en el colectivo de profesionales que no se encuentran activos, como estu- 
El proceso de enfermar de los médicos y sus consecuencias en el profesionalismo - Bárbara Marco-Gómez et al.

diantes, desempleados o jubilados, que quedan desamparados con los recursos actuales.

\section{En conclusión}

Hemos conocido la realidad del médico enfermo en las diferentes etapas profesionales a través de una revisión de la bibliografía existente. Sin embargo, no hemos encontrado apenas publicaciones sobre la situación de los médicos enfermos en países hispanohablantes, por lo que son necesarios más estudios, tanto cuantitativos como cualitativos, que permitan conocer la idiosincrasia del proceso de enfermar de éstos en cada una de sus etapas profesionales, para plantear estrategias de prevención y de mejora en su atención.

\section{Agradecimientos}

This study has been funded by project PI13/02603 (Instituto de Salud Carlos III) and by Fondo Europeo de Desarrollo Regional (FEDER) funds: "Una manera de hacer Europa".

\section{Referencias}

1. Allen H, Traynor M, Kelly D, Smith P. Becoming a patient. En: Understanding sociology in nursing. SAGE Publications Ltd; 2016: 7-10.

2. Parsons T. Illness and the role of physicians: a sociological perspective. American Journal of Orthopsychiatry 1951; 21: 452-460. doi:10.1111/j.1939-0025.1951.tb00003.x

3. Altisent R. La ética del desarrollo profesional continuado en la promoción de la calidad asistencial. Acta Bioethica 2010; 16(2): 155-164.

4. Firth-Cozens J. Intervention to improve physicians' wellbeing and patient care. Soc Sci Med 2001; 52(2): 215-222.

5. Casas M, Gual A, Bruguera E, Arteman A, Padros J. El Programa de Atención Integral al Médico Enfermo (PAIME) del Colegio Oficial de Médicos de Barcelona. Med Clin. 2001; 117: 785-789.

6. Cronin, P, Ryan F, Coughlan M. Undertaking a literature: a step by step approach. British Journal of Nursing 2008; 17: 38-43.

7. Stanton J, Caan W. How many doctors are sick? BMJ 2003; 326: S97.

8. Frank E, Biola H, Burnett CA. Mortality rates and causes among US Physicians. Am J Prev Med 2000; 19: 155-159.

9. O'Hagan J. Doctors and their health. In: O'Hagan J, Richards J, editors. In sickness and in health. $2^{\text {nd }}$ ed. Wellington, NZ: Doctors' Health Advisory Service; 1998: 1-9.

10. Schlincht SM, Gordon IR, Ball RJ, Christie DG. Suicide and related deaths in Victorian doctors. Med J Aust 1990; 153: 528-521.

11. Rosvold EO, Bjertness E. Illness behaviour among Norwegian physicians. Department of General Practice and Community Medicine, University of Oslo, Norway. Scand J Public Health 2002; 30: 125-132.

12. Lindeman S, Laara E, Hakko H, Lonnqvist J. A systematic review on gender-specific suicide mortality in medical doctors. Br J Psichiatry 1996; 168: 274-279.

13. Aasland OG, Ekeberg O, Schweder T. Suicide rates from 1960 to 1989 in Norwegian physicians compared with other educational groups. SocSci Med 2001; 52: 259-265.

14. Toyry S, Rasanen K, Kujala S, Aarimaa M, Juntunen J, Kalimo R, Luhtala R, et al. Self-reported health, illness, and self-care among finnish physicians: a national survey. Arch Fam Med 2000; 9: 1079-1085.

15. Rohlfs I, Arrizabalaga P, Artazcoz L, Borrell C, Fuentes M, Valls C. Salut, estils de vida i condicions de treballdelsmetges $i$ metgesses de Catalunya. Barcelona: Fundació Galatea. Inc; 2007.

16. Iversen A, Rushforth B, Forrest K. How to handle stress and look after your mental health. BMJ 2009; $27:$ b1368.

17. Champbell S, DelvaD. Physician do not heal thyself: Survey of personal health practices among medical residents. Can Fam Physician 2003; 49: 1121-1127.

18. Firth-Cozens J. Depression in doctors. En: Robertson MM, Katona CLE, editors. Depressions and physical illness. New York: John Wiley \& Sons; 1997.

19. Skipper GE, Campbell MD, Dupont RL. Anesthesiologist with substance use disorders: a 5-year outcome study from 
16 state Physician Health Programs. Anesthesia et Analgesia 2009; 109: 891-896.

20. Talbott GD, Martin CA. Treating impaired physicians: fourteen keys to success. Van Med 1986; 113(2): 95-99.

21. Casas M, Gual A, Bruguera E, Arteman A, Padros J. El Programa de Atención Integral al Médico Enfermo (PAIME) del Colegio Oficial de Médicos de Barcelona. Med Clin (Barc). 2001; 117: 785-789.

22. Gallegos KV, Veit FW, Wilson PO, Porter T, Talbott GD. Substance abuse among health professionals. Md Med J. 1988 Mar; 37(3): 191-197.

23. Bruguera M, Guri J, Arteman A, et al. La atención de los médicos hacia el cuidado de su propia salud. Med Clin 2001; 117: 487-494.

24. McCracken CB. A piece of my mind. Intellectualization of drug abuse. JAMA 2010; 303(19): 1894-1895.

25. Roberts LW, Kim JP. Informal health care practices of residents: "Curbside" consultation and self-diagnosis and treatment. Acad Psychiatry 2015; 39: 22-30.

26. Wachtel TJ, Wilcox VL, Moulton AW, Tammaro D, Stein MD. Physicians' utilization of health care. J Gen Intern Med 1995; 10: 261-265.

27. Kay M, Mitchell G, Clavarino A, Doust J. Doctors as patients: a systematic review of doctors' health access and the barriers the experience. BJGP 2008; 58: 501-508.

28. Forsythe M, Calnan M, Wall B. Doctors as patients: postal survey examining consultants and general practitioners adherence to guidelines. BMJ 1999; 519: 605-608.

29. Johnson WDK. Predisposition to emotional distress and psychiatric illness amongst doctors: the role of unconscious and experiential factors. Br J Med Psychol 1991; 64: 317-329.

30. Lawrence J. Stress and the doctor's health. Aust Fam Physician 1996; 25: 1249-1256.

31. Rucinski J. Mentally ill doctors. Br J Hosp Med 1985; 3311: 90-94.

32. Riley GJ. Understanding the stresses and strains of being a doctor. Med J Aust 2004; 181(7): 350-353.

33. O'Connor M, Kelleher C. Do doctors benefit from their profession? A survey of medical practitioners' health promotion and health safety practices. Irish Medical Journal 1998; 91(6): 206-209.

34. Henderson M, Brooks SK, Del Busso L, Chalder T, Harvey SB, Hotopf M, Madan I, Hatch S. Shame! Self-stigmatisation as an obstacle to sick doctors returning to work: a qualitative study. BMJ Open 2012 Oct 15; 2(5). doi: 10.1136/ bmjopen-2012-001776.

35. Davison SK, Schattner PL. Doctors' health-seeking behaviour: a questionnaire survey. Med J Aut 2003; 179: 302-305.

36. Forsythe M, Calnan M, Wall B. Doctors as patients: postal survey examining consultants and general practitioners adherence to guidelines. BMJ 1999; 319: 605-608.

37. Toyry S, Rasanen K, Seuri M, Aarimaa M, Juntunen J, Kujala S, Husman K. Increased personal medication use among Finnish physicians from 1986 to 1997. Br J Gen Pract 2004; 54: 44-46.

38. Kay MP, Mitchell GK, Del Mar CB. Doctors do not adequately look after their own physical health. Med J Aust 2004; 181: 368-370.

39. Hem E, Stokke G, Tyssen R, Gronvold NT, Vaglum P, Ekeberg O. Self-prescribing among young Norwegian doctors: a nine-year follow-up study of a nationwide sample. BMC Med 2005; 3(16):1-7.

40. Firth-Cozens J, Payne R. Stress in health professionals. Chichester: John Wiley et Sons; 1999.

41. Farber NJ, et al. Physicians' willigness to report impaired colleagues. SocSci Med 2005; 61: 1772-1775.

42. Firth-Cozens J. Intervention to improve physicians' wellbeing and patient care. SocSci Med 2001; 52: $215-222$.

43. Lobo Prabhu SM, Molinari VA, Hamilton JD, Lomax JW. The aging physician with cognitive impairement approaches to oversight, prevention and remediation. Am J Geriatr Psychiatry 2009; 17(6): 445-454.

44. Dewa CS, Jacobs P, Thanh NX, Loong D. An estimate of the cost of burnout on early retirement and reduction in clinical hours of practicing physicians in Canada. BMC Health Serv Res. 2014; 14: 254. doi: 10.1186/1472-6963-14-254

45. Aasland OG, Forde R. Impact of feeling responsable for adverse events on doctos' personal and professional lives: the importance of being open to criticism from colleagues. QUalSaf Health Care 2005; 14: 13-17.

46. Gallagher TH, Waterman A, Garbutt J, Kapp J, Chan D, Dunagan C, Fraser V, Levinson W. US and Canadian Physicians' Attitudes and Experiences Regarding Disclosing Errors to Patients. Archives of Internal Medicine 2006; 166: 1605-1611.

47. Waterman AD, Garbutt J, Hazel E, Dunagan WC, Levinson W, Fraser V, et al. The emotional impact of medical errors on practicing physicians in the United States and Canada. The Joint Commission Journal on Quality and Patient Safety. 2007; 33(8): 467-476.

48. Mira JJ, Carrillo I, Lorenzo S, et al. The Aftermath of Adverse Events in Spanish Primary Care and Hospital Health Professionals. BMC Health Service Research 2015; 15: 151. 
El proceso de enfermar de los médicos y sus consecuencias en el profesionalismo - Bárbara Marco-Gómez et al.

49. Wu AW. Medical error: the second victim. BMJ 2000; 320: 726-727.

50. Seys D, Scott S, Wu A, Van Gerven E, Vleugels A, Euwema M, Panella M, Conway J, Sermeus W, Vanhaecht K. Supporting involved health care professionals (second victims) following an adverse health event: a literature review. Int $J$ Nurs Stud. 2013; 50: 678-687.

51. Seys D, Scott SD, Wu AW, Van Gerven E, Vieugels A, Euwema M, Panella M, Conway J, Vanhaecht K. Supporting involved health care professionals (second victims) following an adverse health event: a literature review. International Journal of Nursing Studies. July 30, 2012.

52. Scott SD, Hirschinger LE, Cox KR. Sharing the load: Rescuing the healer after trauma. RN 2008; 71: $38-43$.

53. Scott SD. The second victim phenomenon: A harsh reality of health care professions. Patient Safety Network - Agency for Healthcare research and Quality - U.S. Department of Health and Human Services; 2011. Available at https://psnet. ahrq.gov/perspectives/perspective/102 [Último acceso 21 octubre 2017]

54. Aranaz JM, Mira JJ, Guilabert M, Vitaller J, Herrero JF. Repercussion of adverse events upon the healthcare professionals. A study of the second victims. Trauma Fund MAPFRE 2013; 24(1): 54-60.

55. Bazo Royo MT, Hernández Rodríguez G, et al. Sociología de la Vejez, en Principios de Geriatría y Gerontología. Madrid: McGraw Hill; 2006.

56. Neurgaten BL. Los significados de la edad. Barcelona: Herder; 1996.

57. Sánchez-Candamio M, Del Valle A. Estudio de las necesidades de los profesionales de la Medicina relacionados con la jubilación. Barcelona: Fundación Galatea. Inc; 2006.

58. Silver MP, Williams SA. Reluctance to Retire: A Qualitative Study on Work Identity, Intergenerational Conflict, and Retirement in Academic Medicine. Gerontologist 2016; 00: 1-11.

59. Luce A, Van Zwanenberg T, Firth-Cozens J, Tinwell C. What might encourage later retirement among general practitioners. J Manag Med. 2002; 16(4-5): 303-310.

60. Silver MP, Pang NC, Williams SA. "Why give up something that works so well?”: retirement expectations among academic physicians. Educ Gerontol 2015; 41: 333-347.

61. Gundersen L. Physician burnout. Ann Intern Med 2001; 35: 145-148.

62. Silver MP. Critical reflection on physician retirement. Can Fam Physician 2016; 62: 783-784.

63. Silver MP, Hamilton AD, Biswas A, Warrick NI. A systematic review of physician retirement planning. Human Resources for Health 2016; 14: 67. DOI: 10.1186/s12960-016-0166-z

64. Silver MP, Easty LK. Planning for retirement from medicine: a mixed-methods study. CMAJ Open 2017. Doi: 10.9778/ cmajo.20160133

65. Morales M, López-Ibor JJ. Estrés y adaptación al inicio de la formación médica postgraduada. Actas Luso-Esp. Neurol. Psiquiatr.1995; 23(5): 241-248.

66. Zbieranowski I, Takahashi SG, Verma S, Spadafora SM. Remediation of residents in difficulty: a retrospective 10-year review of the experience of a postgraduate board of examiners. Acad Med. 2013 Jan; 88(1): 111-116. Doi: 10.1097/ ACM.0b013e3182764cb6

67. Ania O, Cuatrecasas G, et al. La salud del MIR: Guia para tutores y profesionales de los centros sanitarios docentes. Barcelona: Fundación Galatea; 2008.

68. Mahmood T. Dealing with trainees in difficulty. Facts Views Vis Obgyn. 2012; 4(1): 18-23.

69. Buddeberg-Fischer B, Klaghofer R, Stamm M, Siegrist J, Buddeberg C. Work stress and reduced health in young physicians: prospective evidence from Swiss residents. Int Arch Occup Environ Health 2008 Oct; 82(1): 31-38. Doi: 10.1007/ s00420-008-0303-7.

70. Programa de Atención Integral al Médico Enfermo: datos de evolución 1998-2014. Madrid: OMC; 2015.

71. Blancafort X, Masachs E, Valero S, Arteman A. Estudio sobre la salud de los residentes de Cataluña. Barcelona: Fundación Galatea; 2009.

72. Baldwin PJ, Dodd M, Wrate RM. Young doctors' health--II. Health and health behaviour. SocSci Med 1997 Jul; 45(1): 41-44.

73. Prins JT, Gazendam-Donofrio SM, Tubben BJ, Van der Heijden FM, Van de Wiel HB, Hoekstra-Weebers JE. Burnout in medical residents: a review. Med Educ 2007 Aug; 41(8): 788-800.

74. Thomas NK. Resident burnout. JAMA 2004; 292: 2880-2889.

75. Lourenção LG, Moscardini AC, Soler ZA. Health and quality of life of medical residents. Rev Assoc Med Bras 2010; 56(1): 81-90.

76. Mata DA, Ramos MA, et al. prevalence of depression and depressive symptoms among resident physicians A systematic review and meta-analysis. JAMA 2015; 314(22): 2373-2383. Doi:10.1001/jama.2015.15845. 
77. Tyssen R, Vaglum P. Mental health problems among young doctors: an updated review of prospective studies. Harv Rev Psychiatry 2002 May-Jun; 10(3): 154-165.

78. Hem E, Stokke G, Tyssen R, et al. Self-prescribing among young Norwegian doctors: a nine-year follow-up study of a nationwide sample. BMC Med 2005; 3: 16

79. Gendel MH, Brooks E, Early SR, Gundersen DC, Dubovsky SL, Dilts SL, Shore JH. Self-prescribed and other informal care provided by physicians: scope, correlations and implications. J Med Ethics 2012 May; 38(5): 294-298. Doi: 10.1136/medethics-2011-100167.

80. Jena AB, Baldwin DC, Daugherty SR, Meltzer DO, Arora VM. Presenteeism among resident physicians. JAMA 2010 Sep 15; 304(11): 1166-1168. Doi: 10.1001/jama.2010.1315

81. Jena AB, Meltzer DO, Press VG, Arora VM. Why physicians work when sick. Arch Intern Med. 2012 Jul 23; 172(14): 1107-1108. Doi: 10.1001/archinternmed.2012.

82. Borenstein DB, Cook K. Impairment prevention in the training years. A new mental health program at UCLA. JAMA 1982; 247: 2700-2703.

83. Borenstein DB. Should physician training centers offer formal psychiatric assistance to house officers? A report on the major findings of a prototype program. Am J Psychiatry 1985 Sep; 142(9): 1053-1057.

84. Toth EL, Collinson K, Ryder C, Goldsand G, Jewell LD. Committee to prevent and remediate stress among house staff at the University of Alberta. CMAJ 1994; 150: 1593-1597.

85. Dabrow SL, Russell S, Ackley K, Anderson E, Fabri PJ. Combating the stress of residency: one school's approach. Acad Med. 2006 May; 81(5):436-439.

86. Levey RE. Sources of stress for residents and recommendations for programs to assist them. Acad Med. 2001; 76: 142150.

87. Hamilton TK, Schweitzer RD. The cost of being perfect: perfectionism and suicide ideation in university students. Aus NZ J Psychiatry 2000; 34: 829-835.

88. Salamero M, Baranda L, Mitjans A, Baillés E, Càmara M, Parramon G, et al. Estudio sobre la salud, estilos de vida y condicionantes académicos de los estudiantes de medicina de Cataluña. Inf y Estud Fund Galatea [Internet]; 2012. Available from: http://www.fgalatea.org/cast/publicacions.php? Up=E

89. Dyrbye LN, Thomas MR, Shanafelt TD. Systematic review of depression, anxiety, and other indicators of psychological distress among US and canadian medical students. Acad Med 2006; 81(4): 354-373.

90. Ishak W, Nikravesh R, Lederer S, Perry R, Ogunyemi D, Bernstein C. Burnout in medical students: A systematic review. Clin Teach 2013; 10(4): 242-245.

91. Dyrbye LN, Thomas MR, Massie FS, Power DV, Eacker A, Harper W, et al. Academia and Clinic. Ann Intern Med [Internet] 2001; 135(7): 507-513. Available from: http://www.councilscienceeditors.org/i4a/pages/index.cfm?pageid=3410 [access: 21 octubre 2017]

92. Woolf K, Cave J, McManus IC, Dacre JE. 'It gives you an understanding you can't get from any book. The relationship between medical students' and doctors' personal illness experiences and their performance: a qualitative and quantitative study. BMC Med Educ 2007; 7: 50.

93. Tomlinson J. Lessons from the "other side": teaching and learning from doctors' illness narratives. BMJ Careers 2014. Available from: http://careers.bmj.com/careers/advice/view-article.html?id=20017843 [access 21 octubre 2017]

94. Svantesson M, Carlsson E, Prenket M, Anderzen-Carlsson A. 'Just so you know, the patient is staff: healthcare professionals' perceptions of caring for healthcare professional-patients. BMJ Open 2016 Jan 19; 6(1): e008507. Doi: 10.1136/bmjopen-2015-008507.

95. Thompson WT, Cupples ME, Sibbett CH, Skan DI, Bradley T. Challenge of culture, conscience, and contract to general practitioners' care of their own health: qualitative study. BMJ 2001; 323(7315): 728-731.

96. Fox F, Harris M, Gordon T, Rodham K, Sutton J, Robinson B, Scott J. What happens when doctors are patients. Qualitative study of GPs. Br J Gen Pract. 2009 Nov; 59(568): 811-818. Doi: 10.3399/bjgp09X472872.

97. American Medical Association's Council on Mental Health: The sick physician: Impairment by psychiatric disorders. JAMA 1973; 223: 684-687.

98. Federation of state physician health programs, $A M A$. Available from: http://www.fsphp.org/ [access 21 oct 2017]

99. CMA: Centre for Phisician Health and wellbeing. Available from: https://www.cma.ca/En/Pages/physician-healthwellness.aspx [access 21 oct 2017]

100. Doctors' health advisory service. Available from: https://ama.com.au/resources/doctors-health [access 21 oct 2017 ].

101. British Medical Association: Doctors health and wellbeing. Available from: http://bma.org.uk/support-at-work/doctors-well-being [access 21 oct 2017].

102. General Medical Council. Good medical practice. London: GMC, 1995. United Kindgdom: Health. Available from: 
El proceso de enfermar de los médicos y sus consecuencias en el profesionalismo - Bárbara Marco-Gómez et al.

http://www.gmc-uk.org/guidance/good_medical_practice/your_health.asp [access 21 oct 2017].

103. Braquehais MD, Bela MJ, Montejo JE, Arteman A, Bruguera E, Casas M. El Programa de Atención Integral al Médico Enfermo de Barcelona: salud mental para una buena praxis. Rev Esp Med Legal 2012; 38(3): 107-112.

104. Braquehais MD, Valero S, Bel MJ, Navarro MC, Matalí JL, Nasillo V et al. Doctors admitted to a Physicians' Health Program: a comparison of self-referrals versus direct referrals. BMJ Open 2014; 4: e005248. Doi: 10.1136/bmjopen-2014-005248.

Recibido: 28 de noviembre de 2017

Aceptado: 30 de enero de 2018 